\title{
Résumé de la déclaration du Comité consultatif national de l'immunisation sur la revaccination au moyen du vaccin antipneumococcique polysaccharidique 23- valents
}

\author{
Quach $C^{1,2}$, au nom du Comité consultatif national de l'immunisation*
}

${ }^{1}$ Coprésidente du CCNI et présidente du groupe de travail sur le pneumocoque, Montréal (Québec)

${ }^{2}$ Centre d'étude sur les vaccins, Centre universitaire de santé McGill, Montréal (Québec)

*Correspondance : naci-ccni@phac-aspc.gc.ca

\section{Résumé}

Contexte : On recommande que les personnes de 2 ans et plus à haut risque de contracter des infections pneumococciques (définies comme celles souffrant d'asplénie fonctionnelle ou anatomique ou d'anémie falciforme; de cirrhose hépatique; d'une néphropathie chronique ou du syndrome néphrotique; d'une infection au $\mathrm{VIH}$ ou d'une immunodéficience liée à une maladie ou une thérapie) reçoivent une dose de rappel unique du vaccin antipneumococcique polysaccharidique 23-valents (Pneu-P-23); on fait également des recommandations sur l'administration du vaccin conjugué antipneumococcique 13-valents (Pneu-C-13) selon l'âge et le risque. Les adultes âgés de 65 ans et plus sont également considérés comme étant à haut risque pour les infections pneumococciques.

Objectif : Déterminer le délai optimal entre la vaccination initiale au Pneu-P-23 et les doses de rappel subséquentes pour protéger contre les infections pneumococciques chez les personnes à haut risque de les contracter.

Méthodologie : Le Comité consultatif national de l'immunisation (CCNI) a réalisé une revue systématique de la littérature portant sur les doses de rappel du vaccin antipneumococcique chez les personnes à haut risque de contracter des infections pneumococciques. Le CCNI a examiné les preuves en considérant la population cible, l'innocuité, l'immunogénicité, l'efficacité potentielle et l'efficacité réelle des vaccins, les calendriers vaccinaux et d'autres aspects de la stratégie générale d'immunisation, puis a approuvé trois recommandations spécifiques.

Résultats : Pour toutes les personnes âgées de 2 ans et plus à haut risque de contracter des infections pneumococciques qui ont reçu une dose de Pneu-P-23, la revaccination avec une seconde dose de Pneu-P-23 devrait être offerte cinq ans après la dose initiale. On devrait également leur avoir administré antérieurement le vaccin conjugué antipneumococcique 13-valents à des doses adaptées à l'âge. Les preuves sont actuellement insuffisantes pour déterminer de façon optimale le calendrier et le nombre de doses de rappel de Pneu-P-23 chez les adultes à haut risque. On recommande actuellement une dose de rappel unique de Pneu-P-23 chez les personnes à haut risque de contracter des infections pneumococciques, cinq ans après la dose précédente. Étant donné le risque accru de contracter des infections pneumococciques chez les adultes de 65 ans et plus et le déclin rapide d'anticorps suite à l'administration du Pneu-P-23, tout le monde devrait recevoir une dose de Pneu$\mathrm{P}-23$ à l'âge de 65 ans, au moins cinq ans après la dose précédente de Pneu-P-23. Aucune dose de rappel additionnelle n'est actuellement recommandée pour ce groupe d'âge, si aucune condition médicale ne met la personne à haut risque de contracter des infections pneumococciques.

Conclusion : La nouvelle série complète de recommandations actuelles pour les vaccins antipneumococciques sera publiée dans la mise à jour du chapitre sur le pneumocoque dans le Guide canadien d'immunisation dans un avenir rapproché. 


\section{Introduction}

On recommande que les personnes de 2 ans et plus à haut risque de contracter des infections pneumococciques (définies comme celles souffrant d'asplénie fonctionnelle ou anatomique ou d'anémie falciforme; de cirrhose hépatique; d'une néphropathie chronique ou d'un syndrome néphrotique; d'une infection au VIH ou d'une immunodéficience liée à une maladie ou une thérapie) reçoivent une dose de rappel unique du vaccin antipneumococcique polysaccharidique 23-valents (Pneu-P-23); on fait également des recommandations sur l'administration du vaccin conjugué antipneumococcique 13-valents (Pneu-C-13) selon l'âge et le risque. Les adultes de 65 ans et plus sont également considérés comme étant à haut risque de contracter des infections pneumococciques, sans égard à leur condition médicale.

Le Comité consultatif national de l'immunisation (CCNI) donne des conseils médicaux, scientifiques et de santé publique sur l'immunisation à l'Agence de la santé publique du Canada (l'Agence) de façon continue et opportune. Le CCNI a entrepris un examen systématique pour déterminer le délai optimal entre la vaccination initiale au Pneu-P-23 et les doses de rappel subséquentes chez les personnes à haut risque de contracter des infections pneumococciques.

\section{Méthodologie}

Le CCNI a réalisé une revue systématique des preuves en considérant la population cible, l'innocuité, l'immunogénicité, l'efficacité potentielle et l'efficacité réelle des vaccins, les calendriers vaccinaux et d'autres aspects de la stratégie générale d'immunisation, puis a élaboré et approuvé trois recommandations spécifiques.

\section{Résultats}

Dix études ont été recensées au total pour les données sur l'immunogénicité. L'innocuité de la revaccination au Pneu-P-23 a été évaluée dans huit de ces études. Dans toutes les études recensées, la revaccination de ceux à haut risque de contracter des infections pneumococciques cinq ans après une vaccination initiale au Pneu-P-23 a démontré une réponse immunitaire accrue et un profil d'innocuité acceptable. Les personnes ayant reçu une ou deux doses de Pneu-P-23 avant l'âge de 65 ans ont démontré une bonne réaction immunitaire à une dose de rappel de Pneu-P-23, lorsque celle-ci avait été administrée à 65 ans ou plus. II y a peu de données probantes qui laissent croire qu'une hyporéactivité survient après une dose de rappel additionnelle de Pneu-P-23. Un rapport complet de la revue systématique est disponible (1).

Les recommandations suivantes ont été élaborées et approuvées par le CCNI.

\section{Recommandations actualisées du CCNI sur le vaccin Pneu-P-23 (2)}

\section{Recommandation 1}

Pour toutes les personnes âgées de 2 ans et plus à haut risque de contracter des infections pneumococciques (asplénie fonctionnelle ou anatomique ou anémie falciforme; cirrhose hépatique; néphropathie chronique ou syndrome néphrotique; infection au $\mathrm{VIH}$ ou immunodéficience liée à une maladie ou une thérapie) qui ont reçu une dose de Pneu-P-23, la revaccination avec une seconde dose de Pneu-P-23 devrait être offerte cinq ans après la dose initiale. Ceci représente un changement par rapport à la recommandation antérieure selon laquelle les enfants qui recevaient leur première dose de Pneu-P-23 à l'âge de 10 ans ou moins devraient recevoir la seconde dose trois ans plus tard. Cette modification se fonde sur l'absence de preuves à l'appui du calendrier sur trois ans de la dose de rappel chez les enfants et sur l'utilisation universelle de Pneu-C-13 chez les enfants qui a contribué à une diminution marquée de l'incidence des infections pneumococciques. La revaccination unique cinq ans après la vaccination initiale permet d'harmoniser les calendriers pédiatriques et adultes pour ceux présentant un risque élevé de contracter des infections pneumococciques. Les personnes à haut risque auraient dû également recevoir des doses adaptées à leur âge du vaccin conjugué antipneumococcique 13-valents; le vaccin conjugué antipneumococcique 13-valents devrait être administré en premier, suivi du Pneu-P-23 huit semaines plus tard. 


\section{Recommandation 2}

Les preuves sont actuellement insuffisantes pour déterminer de façon optimale le calendrier et le nombre de doses de rappel de Pneu-P-23 chez les adultes à haut risque (c.-à-d., ceux souffrant d'asplénie fonctionnelle ou anatomique ou d'anémie falciforme; de cirrhose hépatique; d'une néphropathie chronique ou d'un syndrome néphrotique; d'une infection au VIH ou d'une immunodéficience liée à une maladie ou une thérapie). On recommande actuellement une dose de rappel unique de Pneu-P-23 chez les personnes à haut risque de contracter des infections pneumococciques, cinq ans après la dose précédente.

\section{Recommandation 3}

Étant donné le risque accru de contracter des infections pneumococciques chez les adultes de 65 ans et plus et le déclin rapide d'anticorps à la suite de l'administration du Pneu-P-23, tout le monde devrait recevoir une dose de Pneu-P-23 à l'âge de 65 ans, pourvu que la dose précédente remonte à au moins cinq ans. Les études recensées pour appuyer cette déclaration actualisée ont toutes administré une dose de Pneu-P-23 aux personnes de 65 ans et plus, sans égard à leur histoire de vaccination antérieure. Aucune dose de rappel additionnelle n'est actuellement recommandée pour les plus de 65 ans qui n'ont pas une autre condition médicale sous-jacente qui les mettrait à haut risque de contracter des infections pneumococciques.

\section{Conclusion}

La nouvelle série complète de recommandations actuelles sur les vaccins antipneumococciques sera publiée dans la mise à jour du chapitre sur le pneumocoque du Guide canadien d'immunisation dans un avenir rapproché (3). Les priorités en matière de surveillance et de recherche sont comme suit : une surveillance accrue qui inclut les personnes à haut risque et qui permettrait d'obtenir l'incidence des infections pneumococciques stratifiée par facteurs de risque et sérotypes chez les 65 ans et plus; des études d'efficacité et de couverture vaccinale du Pneu-P-23 chez les patients à haut risque et les plus de 65 ans; et des études épidémiologiques de maladies non invasives, telles que la pneumonie acquise en communauté dans tous les groupes d'âge ou l'otite moyenne aiguë causée par S. pneumoniae chez les enfants. Pour étayer les recommandations sur les doses additionnelles (p. ex. plus d'une dose de rappel), d'autres études sont nécessaires pour comprendre comment le système immunitaire réagit à des doses additionnelles de Pneu-P-23.

\section{Remerciements}

L'auteure voudrait remercier les membres extrêmement dévoués du CCNI et du groupe de travail du CCNI sur le pneumocoque.

\section{Conflit d'intérêts}

Aucun

\section{Références}

(1) Caya CA, Boikos C, Desai S, Quach C. Dosing regimen of the 23-valent pneumococcal vaccination: A systematic review. Vaccine. 2015 Mar 10;33(11):1302-12. doi: 10.1016/j.vaccine.2015.01.060. Epub 2015 Feb 3.

(2) National Advisory Committee on Immunization (NACl). Re-Immunization with Polysaccharide 23-Valent Pneumococcal Vaccine (Pneu-P-23): An Advisory Committee Statement (ACS). 2015 April http://publications.gc.ca/site/eng/9.629954/publication.html (disponible en français http://publications.gc.ca/site/eng/9.677547/publication.html.

(3) National Advisory Committee on Immunization (NACl). Canadian Immunization Guide. 2015. http://www.phac-aspc.gc.ca/publicat/cig-gci/p04-pneu-eng.php. (disponible en français: http://www.phac-aspc.gc.ca/publicat/cig-gci/p04-pneu-fra.php). 